\title{
EVALUACIÓN DE LA RESISTENCIA DEL SUELO EN CASANARE, COLOMBIA
}

\section{María Del Carmen Fuentes Fuentes}

Grupo de Investigación INGEOFISICA; Universidad Pedagógica y Tecnológica de Colombia. Email: marcaf_geo@yahoo.com

(Recibido: el 27 de Febrero de 2013 y aceptado 22 de Octubre de 2013)

Resumen: El presente artículo contiene los resultados obtenidos durante la interpretación de los datos de resistencia del suelo, correspondientes a los trabajos realizados en la ciudad de Monterrey, Casanare, Colombia.

El objetivo principal de esta investigación, estuvo dado en encontrar el punto óptimo donde sería ubicada la toma a tierra de una subestación, que consta de un sistema electrónico o planta generadora o transmisora de radiofrecuencia.

En el trabajo se presenta una descripción del método geofísico de corrientes telúricas, la metodología y los resultados obtenidos a partir de 21 puntos de mediciones, en una red regular.

Se presenta el mapa de resistividades y el mapa de isolíneas del área que permitieron diferenciar las diferentes zonas.

Palabras Claves: Geofisica, Potencial espontáneo, campo eléctrico natural.

\section{INTRODUCCIÓN}

La investigación que se presenta, forma parte de numerosos proyectos que se han realizado por integrantes del grupo de investigación INGEOFISICA, en coordinación con la empresa IGEOSOFTMINE LTDA, con el fin de investigar el comportamiento de las propiedades geoeléctricas del subsuelo, logrando incrementar los estudios geofísicos en diferentes regiones de Boyacá, Casanare, etc., donde no solo se ha empleado el Sondeo Eléctrico Vertical, sino otros métodos menos mencionados y que son de gran aplicación y ofrecen resultados satisfactorios.

El Departamento del Casanare está situado en el oriente del país la región de la Orinoquía, localizado entre los $04^{\circ} 17^{\prime} 25^{\prime \prime}$ y $06^{\circ} 20^{\prime} 45^{\prime}$ de latitud norte y los 69 $50^{\prime} 22^{\prime \prime}$ y $73^{\circ} 04^{\prime} 33^{\prime \prime}$ de longitud oeste. Cuenta con una superficie de $44.640 \mathrm{~km}^{2}$ lo que representa el $3.91 \%$ del territorio nacional. Limita por el Norte con el río Casanare, que lo separa del departamento de Arauca; por el Este con el río Meta que lo separa del departamento de Vichada; por el Sur con los ríos Upía y Meta, el último de los cuales los separa del departamento del Meta, y por el Oeste con los departamentos de Boyacá y Cundinamarca (ver figura 1).
Abstract: The this paper contains the results obtained during the interpretation of soil resistivity data, corresponding to the work performed in the city of Monterrey, Casanare, Colombia.

The main objective of this research was given to find the optimal point where the grounding of a substation, which consists of an electronic system or plant generator or transmitter of radio frequency would be located.

The paper presents a description of geophysical method of telluric currents, the methodology and the results obtained from 21 points of measurements, in a regular network.

It is the resistivity map and map of isolines of the area allowing you to differentiate between different areas.

Key words: Geophysics, spontaneous potential, field electric natural. 




Foto 1. Depósito coluvial.

Teoría y métodos.

Durante esta investigación, se empleó la siguiente metodología:

- Diseño de la malla y distribución de los puntos de registro sobre el plano de diseño de obras.

- Localización de las coordenadas de los puntos de registro con GPS.

- Toma de los datos de resistividad para cada punto.

- Elaboración, representación e interpretación de datos.

- Confección del informe final.

Siempre que se realice un estudio de estas características, se debe tener en cuenta que la resistividad del terreno varía ampliamente a lo largo y ancho del globo terrestre, estando determinada por:

- Sales solubles

- Composición propia del terreno

- Estratigrafía

- Granulometría

- Estado higrométrico

- Temperatura

- Compactación

En la toma de mediciones, se empleó un terrómetro (llamado en otros países: telurómetro) o Megger de tierras de tres terminales.

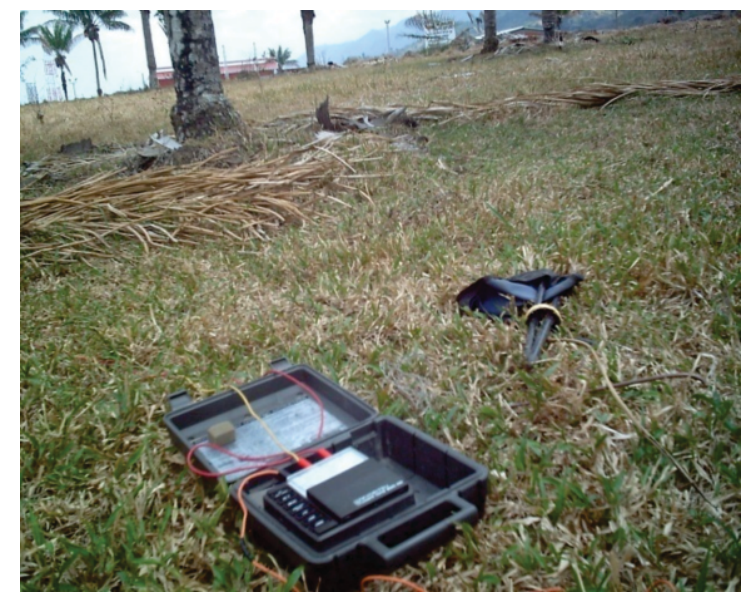

Foto 2. Telurómetro KYORITSU.

El sistema de electrodos se coloca de la forma mostrada en la figura 2.

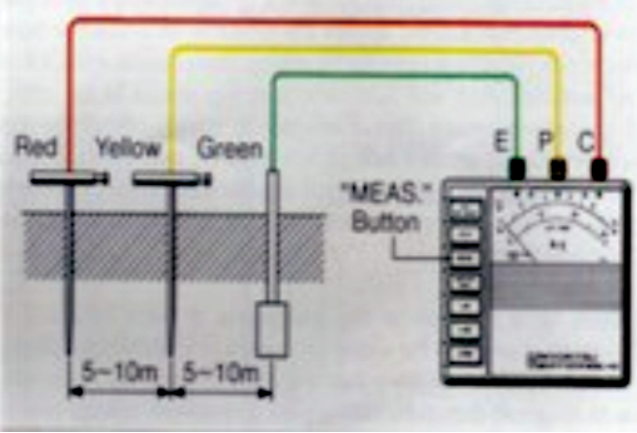

Figura 2. Forma del dispositivo de electrodos.

El procedimiento empleado consistió en:

Inicialmente, se excava un hueco de poca profundidad para instalar el electrodo $\mathrm{E}$, que funciona como masa, de esta manera descartamos resistividades fuera de rango, seguidamente, colocamos dos electrodos de potencial uno a 12.5 metros con referencia al punto de medición y el otro a 25 metros, finalmente se procede a la toma de la lectura, realizando mediciones ortogonales las cuales nos permiten obtener un promedio representativo del valor de la resistividad.

\section{RESULTADOS}

A continuación se exponen los resultados obtenidos; en primer lugar, la tabla de datos obtenida, luego el mapa de resistividades y el mapa en $2 \mathrm{D}$ (ver figuras 3 y 4 ).

Tabla 1. Datos obtenidos.

\begin{tabular}{|c|c|c|c|c|}
\hline$\square E$ & 䍡昌 & 希 湭 & $n \infty$ & R? \\
\hline$=$ & A25 & & & \\
\hline & $\overline{\mathbf{A}}$ & B & C & $\bar{D}$ \\
\hline 1 & 1129164 & 1033846 & 38 & 523.1 \\
\hline 2 & 1129149 & 1033856 & 27 & 522.2 \\
\hline 3 & 1129123 & 1033866 & 43 & 520.9 \\
\hline 4 & 1129109 & 1033878 & 12 & 520 \\
\hline 5 & 1129094 & 1033857 & 9 & 520 \\
\hline 6 & 1129108 & 1033843 & 50 & 521 \\
\hline 7 & 1129128 & 1033828 & 63 & 522.4 \\
\hline 8 & 1129130 & 1033788 & 28 & 523 \\
\hline 9 & 1129112 & 1033804 & 74 & 522.3 \\
\hline 10 & 1129088 & 1033815 & 78 & 521 \\
\hline 11 & 1129070 & 1033826 & 10 & 519.8 \\
\hline 12 & 1129037 & 1033801 & 16 & 519.7 \\
\hline 13 & 1129059 & 1033795 & 36 & 520 \\
\hline 14 & 1129084 & 1033770 & 81 & 521.8 \\
\hline 15 & 1129103 & 1033732 & 35 & 523 \\
\hline 16 & 1129054 & 1033751 & 14 & 521.5 \\
\hline 17 & 1129031 & 1033762 & 27 & 521 \\
\hline 18 & 1129007 & 1033780 & 56 & 519.8 \\
\hline 19 & 1129986 & 1033739 & 24 & 520.5 \\
\hline 20 & 1129019 & 1033718 & 46 & 522 \\
\hline 21 & 1129053 & 1033685 & 58 & 525 \\
\hline
\end{tabular}

Donde:

$\mathbf{A}=$ Coordenada $\mathrm{E}$

$\mathbf{B}=$ Coordenada $\mathrm{N}$

$\mathbf{C}=$ Resistencia promedio del terreno $=\Omega$

D = Altitud (m.s.n.m.)

Coordenadas con alturas corregidas y datos de resistividad obtenidos. 
En el mapa de resistividades y el mapa de tendencias resistivas, se observa que los mayores valores de resistividades se aprecian en la parte noroeste del área, los cuales son asociados con la presencia del depósito matriz-soportado (arenoarcilloso) de origen coluvial compuesto por cantos, gravas y bloques de areniscas que superan los $2 \mathrm{~m}$ de espesor, de muy buena permeabilidad, de ahí que las resistividades oscilen sobre los 50 ?, pero que se diferencian de la parte centro y sur del área donde se observan anomalías suaves de muy baja resistividad (10-50 ?), correspondientes a la zona de areniscas saturadas del depósito de muy alta conductividad.

Aunque la mayor parte del área es de baja resistividad, para la instalación de la subestación, se recomienda que se realice el tratamiento del terreno empleando productos que bajen aún más el valor de la resistencia obtenido, para evitar que existan influencias negativas sobre los sistemas electrónicos generadores de radio frecuencia.

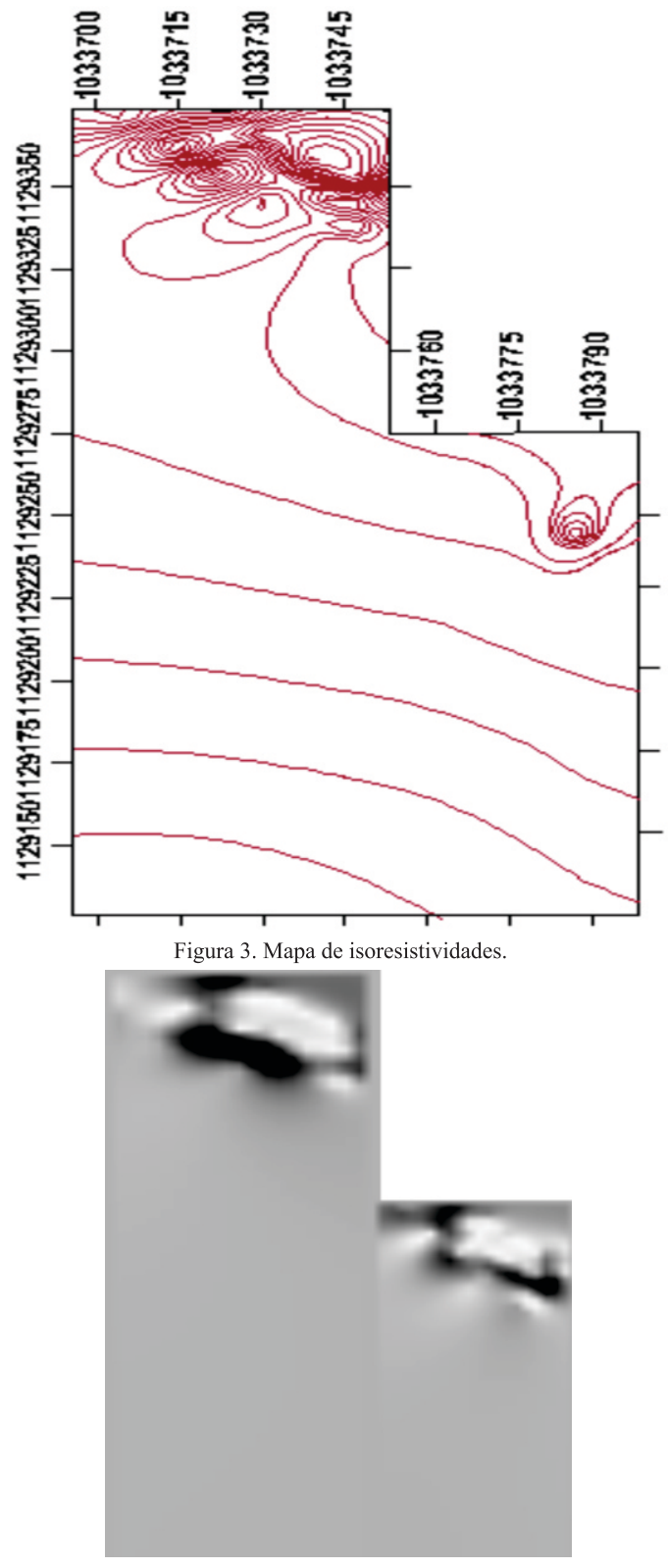

Figura 4. Mapa en 3D, de tendencias resistivas.

\section{CONCLUSIONES}

$\checkmark$ Para diseñar un sistema de tierras de gran tamaño, es aconsejable encontrar el área de más baja resistividad para lograr una instalación más rápida y económica.

$\checkmark$ El perfil de la resistividad del suelo determina el valor de la resistencia a tierra y la profundidad del sistema de puesta a tierra.

$\checkmark$ La resistividad puede ser empleada para indicar el grado de corrosión de tuberías subterráneas. Generalmente, los lugares con resistividad baja tienden a incrementar la corrosión.

$\checkmark$ Según los resultados obtenidos en el presente estudio, la tendencia de resistencias bajas se localizan hacia los puntos de medición número $3,4,5$ y alternativamente el 12 , por lo que se recomienda proyectar la instalación de polos a tierra hacia estos sectores.

$\checkmark$ Se recomienda realizar tratamiento del terreno con productos especiales para bajar la resistencia del terreno por debajo $5 \Omega$.

\section{REFERENCIAS}

IGEOSOFTMINE S.A., 2009- 2011, Informe Métodos de prospección y exploración de aguas subterráneas en Boyacá, Colombia, 200 págs.

GEOFÍSICA I MONTES, Orestes. Ed. Pueblo y Educación, Habana cuba 1978.

Chang J. L; y otros. (2004). Aplicación de técnicas geofísicas en la evaluación de riesgos geológicos en la comunidad.

Resúmenes de la VII Jornada Científica de la SCG, Filial del Centro. Santa Clara.

Dolphin, L.T. (1981). Geophysical methods for archaeological surveys in Israel: Stanford Research Intemational, Menlo Park, Calif., December 1981, 7 pp.

Fajklewicz, A., Glinski, A., and Sliz, J. (1982). Some applications of the underground tower gravity vertical gradient- Geophysics 47, 1688-1692.

Foster, E.J. (1968). Further developments of the pulsed induction metal detector. Prospezioni

Archeologiche 3, 95-99. 